Copyright $@$ ACM, 2021. This is the author's version of the work. It is posted here by permission of $A C M$ for your personal use. Not for redistribution. The definitive version was published in CHI EA '21: Extended Abstracts of the $2021 \mathrm{CHI}$ Conference on Human Factors in Computing Systems, 08 May 2021.

https://doi.org/10.1145/3411763.3451727

\title{
Design Heuristics for Artificial Intelligence: Inspirational Design Stimuli for Supporting UX Designers in Generating Al-Powered Ideas
}

\section{Accepted for 2021 ACM CHI Virtual Conference on Human Factors in Computing Systems 8 - 13 May 2021 Yokohama Japan (Virtual)}

\author{
XIAONENG JIN* \\ College of Design and Innovation, Tongji University, Shanghai, China, xiaoneng_jin@hotmail.com \\ Mark Evans \\ School of Design and Creative Arts, Loughborough University, Loughborough, UK, m.a.evans@lboro.ac.uk \\ Hua Dong \\ Brunel Design School, Brunel University London, London, UK, hua.dong@ brunel.ac.uk \\ Anqi Yao \\ Alibaba Group, Hangzhou, China, anqi.yao@alibaba-inc.com
}

Artificial Intelligence (AI) will provide novel User Experience (UX) solutions if UX designers understand how AI can be best utilized. They need to understand AI capabilities and envisage potential applications. To aid the ideation processes, design heuristics for AI are needed to support UX designers in the conceptual design stage. Forty design heuristics were extracted from 1,755 granted AI patents through a four-step process. The feasibility of the heuristics was verified with two AI-powered case studies: a smart canteen and an online smart shopping system. Case studies suggest that AI design heuristics can be used as design stimuli in the early conceptual design phase to support practitioners in exploring a larger design space for the generation of AI-powered ideas.

CCS CONCEPTS: $\bullet$ Human-centered computing $\rightarrow$ Interaction design $\rightarrow$ Interaction design process and methods

Additional Keywords and Phrases: Artificial intelligence; Design heuristics; UX design; Creativity; Design tools

\section{Introduction}

Artificial Intelligence (AI) can enhance life in a number of fields e.g., transportation, home/service robots, healthcare, education, public safety and security [62]. AI is a foundational technology that can boost competitiveness, increase productivity, protect national security and help resolve societal challenges [17]. These unique features/capabilities have been identified by designers with interest in incorporating the advantages of $\mathrm{AI}$ in their designs $[18,71]$. However, research suggests that practitioners are challenged in their understanding of AI capabilities and capacity to envisage novel and implementable solutions for a given UX problem [18, 72].

To support practitioners, AI tools, methods and aids $[15,3,26,74]$ have been developed. Unfortunately, these methods predominately support the later phases of the design process (i.e. the evaluation phase). Few studies have been conducted to explore ways in which practitioners might generate a greater number of novel and diverse concepts for the AI domain in the early conceptual design phase.

The ability to take a problem and generate multiple, varied solutions that can lead to new creative outcomes is often referred to as concept generation or ideation [59]. Ideation originally determines the type of design and plays an important role in the development of novel concepts and, ultimately, potential for business success [30]. Generating a diverse range of ideas can be challenging as designers become 'fixated' whereby attention becomes focused on a single past example or one new idea [34, 66]. UX practitioners meet design fixation challenges in generating AI-powered ideas beyond automation, recommenders, and reminders [71].

In an attempt to increase concept generation for AI enabled UX concepts our research question is: How might practitioners be supported in understanding the potential of AI to facilitate the generation of novel and diverse concepts in the early conceptual design phase?

\footnotetext{
* Corresponding author
} 
This paper focuses on developing 'creative tools' for AI. Creative tools are required to aid the designer to produce more 'creative' ideas in short periods [30]. 'Design heuristics (DHS)' are a cognitive 'shortcuts' that point toward useful design patterns to reduce search time [23]. DHS as a tool can help boost creativity in the early conceptual design phases [73]. DHS has not only been developed to support general design tools (e.g., TRIZ[24], SCAMPER[19], DHS77[73]) but also were developed for specific fields (e.g., DHS for additive manufacturing, DHS for digital design) [10,35]. AI is becoming a key technology to benefit many areas [62] however, there are no design heuristics for AI applications.

The aim of this study is to systematically analyze AI-related applications and technologies based on granted patent documents from 2008 to 2020 and propose Design Heuristics for AI (AIDHs). Our study contributes to the lack of existing knowledge of DHS in the digital era [36] through three main contributions: 1) development of the tool 40 AI DHS by systematically analyzing 1,755 granted patents in AI domain; 2) a proposal of a new method based on structural big data for extracting DHS; 3) taking AI from uncanny to explainable to support design ideation.

\section{Background}

\subsection{Artificial Intelligence as new design material}

Technology tends to evolve from an elite niche to a mainstream tool and AI is undergoing a similar transformation [44]. AI has realized as a new design opportunity in recent years, especially in the UX domain [18, 71]. Whilst AI emerged in the 1950's, there has been increasing interest with increases in data and computing power [7] and can contribute to a broad number of fields e.g., transportation, home/service robots, healthcare, education, public safety and security, etc [62]. AI technology is now becoming mature and accessible and as we enter what has been identified as the age of deployed AI [44]. The key issue is no longer how AI technology works, but what useful work it can do [44].

\subsection{Challenges for designing AI applications}

Yang et al. [72] explored why AI is uniquely difficult to design for i.e. it is difficult to articulate what AI can/cannot do. There are challenges in understanding AI and its capabilities in not knowing how to purposefully use AI for the design problem at hand [18]. Designers' understanding of AI is limited, making it difficult to ideate, sketch, and prototype AI concepts [18]. Generating a diverse range of ideas is challenging as designers can become 'fixated' [34, 66]. Despite the emphasis on creative exploration, designers have been shown to experience limitations when attempting to generate diverse concepts [14]. For example, practitioners meet design fixation challenges in generating AI-powered ideas beyond automation, recommenders, and reminders [71].

\subsection{Existing Al design tools}

Developing tools and aids for supporting design for AI has become a popular topic over recent years. Google developed AI guidance for practitioners [26]; Amershi et al. [3] developed 18 guidelines for Human-AI Interaction; Corbett et al. [15] propose 'Interactive Machine Learning Heuristics Evaluation'; and Zhou et al. [74] put forward a design method called Material Lifecycle Thinking (MLT) that considers ML as a design material with its entire lifecycle. However, these methods are predominately useful in the later phase of the development process. There are a lack of tools to support AI-powered UX design in the early conceptual design phase where practitioners face challenges in understanding AI capabilities and imagining novel AI solutions for given UX issues [72]. Ideation determines the type of design and plays an important role in the development of novel concepts and business success [30]. However, few studies have been conducted to support practitioners in the generation of novel and diverse concepts for the AI domain in the conceptual design phase.

\subsection{Analogical reasoning and design heuristics in design research}

Analogical reasoning is the process by which information from a source is applied to a target through the connection of relationships or representations [25, 45]. Design researchers have demonstrated that analogies can help promote the generation of additional solutions, or solutions with positive characteristics such as novelty [40,22, 48]. Design Heuristics (DHS) are a context-dependent directive based on intuition, tacit knowledge or experiential understanding which provides design process direction to increase the chances of reaching a satisfactory, but not necessarily optimal, solution [23]. DHS are evidenced to help generate ideas effectively in the conceptual design phase and play an important role in addressing issues of design fixation [73]. Howard [29] suggests that design tools can be categorized as three domains - creative-analysis tools, thinking tools, stimuli tools with DHS being categorized as a stimuli tool. Different DHS have been developed for different purposes, such as DHS for additive manufacturing [10], DHS for assistive (one-handed) products [32] and DHS for digital design [35]. Support of DHS for AI is limited.

\subsection{Patent datasets}

Patent documents are a rich source of technological and commercial information and represent a comprehensive research resource [56]. Patent must be both useful and novel [48] and, as such, have been considered sources of analogies and concepts that can lead to 
innovative solutions [48]. TRIZ [33], a widely used DHS for Engineering Innovation, is also based on patent abstract analysis. This inspired us to look at existing AI patents available in English and Chinese, with the scope limited by the authors' language skills. It was found that there has been a steady increase in the numbers of granted AI patents in America, Europe and China since 2008. Interestingly, in 2019 the figures suggest a dramatic increase. The data indicates that AI technology applications are becoming mature and that this technology will enter a high-speed development period in the near future. The large number of granted patents provides an opportunity to extract DHS through patent-based analysis which is a common method to investigate modern technology dynamics and recent trends in scientific development [31].

\subsection{Research opportunity}

Although tools, methods and aids for designing AI have been proposed [15, 3, 26, 74], few design stimuli tools were developed for AI, especially in the design heuristics domain. Technical advances have triggered an opportunity for design innovation to generate a wealth of new products [18]. A challenges for designing AI [72] are the limited timely innovations in AI applications. Design has the capacity to engage in the innovation processes in AI applications as the design discipline is adept in discovering users' actual needs and generating creative solutions through design thinking/methods [16]. Tools and techniques are needed to support practitioners in generating diverse and innovative AI-powered ideas. By analyzing AI-related patents, a series of AIDHs can be extracted to meet the needs of the fastdeveloping digital era [24].

\section{Research Methods}

To develop a design stimuli tool (Design Heuristic) with a focus on AI, a four-step process was adopted as identified in in Figure 1.

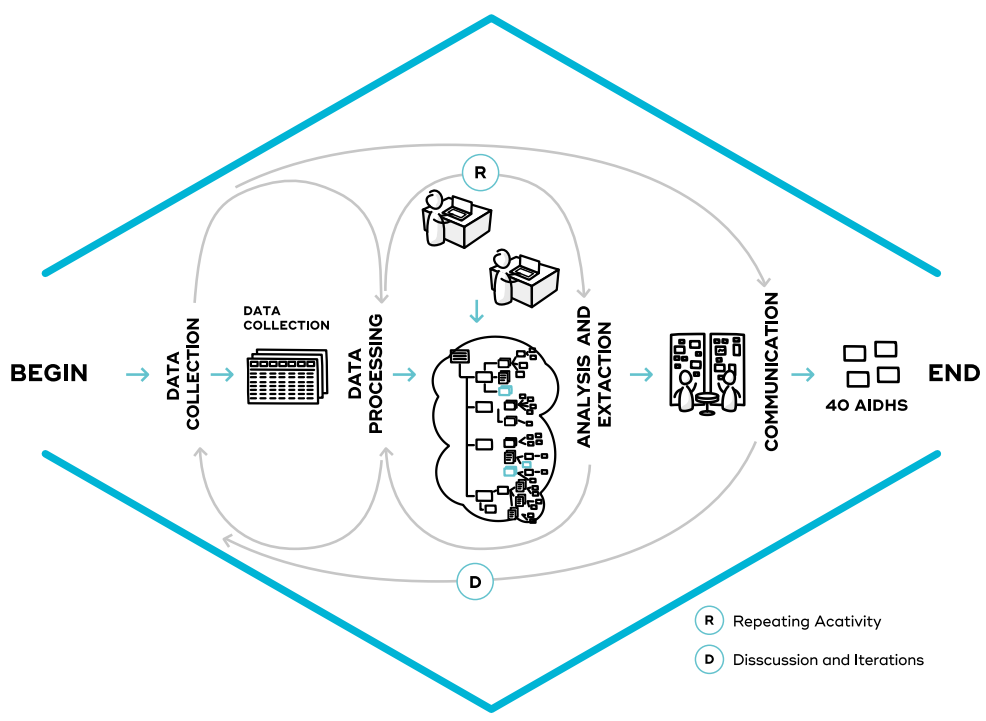

Figure 1 The framework of extracting design heuristics.

\subsection{Phase 1: Data collection}

The patent data was collected from Derwent Innovation, the world's most comprehensive database of enhanced patent information (https://www.derwentinnovation.com/login/) and exported to Microsoft Excel files for further analysis. Table 1 shows the search strategy. To extract highly innovative DHS, the patents of the Utility Model and Pending Patents were excluded. Only patents from the United States, China and the Europe were included as these countries and regions lead AI technology and marketing and English and Chinese were accessible languages to the authors. 'Artificial Intelligence' was used as the keyword in searching the Title/Abstract of all patent documents. The publication dates included the period from 1st January 2008 to 1 st January 2020. In total, 2,062 granted patents from Derwent Innovation were included. In the Microsoft Excel file, data was organized as Patent Name, Patent Title, Abstract, and Assignee/Applicant. Further filtering (e.g. eliminating 'dead' and indeterminate patents) results in 1,755 patents.

\section{Table 1 Search Strategy}

\author{
Search Strategy \\ $\mathrm{TAB}=($ artificial intelligence $)$ AND DP $>=(20080101)$ \\ AND $\mathrm{DP}<=(20200101)$;
}

\section{Patent Collections}

Collection: DWPI, CN Grant, EP Grant,

US Grant (Applications and Utility Models are excluded) 
(TAB=Title/Abstract; DP=Publication Date $)$

Record(s) found out

Filtering the Alive patents

(Dead and Indeterminate are excluded)
$\mathrm{CN}=\mathrm{China}, \mathrm{EP}=$ Europe, $\mathrm{US}=$ United States

2,062

1,755 (Application Language: EN, ZH)

$\mathrm{EN}=$ English, $\mathrm{ZH}=$ Chinese

\subsection{Phase 2: Data processing}

The Word Frequency was utilized which was computed (including stemmed words) using QSR NVivo 12® [8, 20]. The word frequency was exported to a Microsoft Word file for further analysis.

\subsection{Phase 3: Analysis and extraction}

Two researchers undertook the data extraction processes with each analyzing and extracting the AI design heuristics individually.

1. Analyzing high-frequency words. By reviewing every high-frequency word that appeared in more than three patent titles individually, the two researchers selected words independently and highlighted them in Microsoft Word. In this phase, 46 potential words were identified as the initial Design heuristics (DHS). Appendix A.1 shows preliminary DHS extraction based on the Word Frequency and the researchers' analysis.

2. Contrasting the details of patent documents and potential AIDHs. 46 potential DHS nodes were created by QSR NVivo $12{ }^{\circledR}$ after which the researchers utilized every potential DHS as a keyword to track the patent title for further contrasting. Every tracked patents' title was then reviewed to identify if these patents were highly relevant to the potential DHS and the patent's title added to the DHS node one by one.

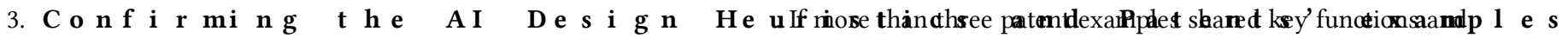
features, design heuristics were confirmed based on these node groups.

\subsection{Phase 4: Communication}

In this phase, the two researchers entered discussions to reach a consensus. For example, some DHS were similar such as analyzing and analysis, automated and automatic, decision and determining, identification and identifying/recognition, and prediction/forecasting so these were combined.

\section{Results}

Table 2 shows the list of extracted design heuristics for AI accompanied by examples and divided into thematic categories. The AI design heuristics were general (i.e. Analogical Reasoning) so that the imagination and creativity of designers would not be constrained by excessive detail [24]. To make the design heuristics as easy to understand and remember as possible, 40 AIDHS are organized via four categories - decision making, personalization, productivity, security.

Table 2 Extracted design heuristics for AI accompanied by examples and divided into thematic categories

\begin{tabular}{|c|c|c|}
\hline No. & DHS & Examples (Title) \\
\hline A & \multicolumn{2}{|c|}{ DECISION MAKING } \\
\hline 1 & Analyzing & Artificial intelligence based vehicle dashboard analysis [4] \\
\hline 2 & Determining & Method for determining acceptability of proposed color solution using an artificial intelligence model [43] \\
\hline 3 & Diagnosis & Artificial intelligence-based system and methods for corneal diagnosis [1] \\
\hline 4 & Evaluation & A video evaluation recommendation system [27] \\
\hline 5 & Judging & Based on artificial intelligence of the intelligent robot of multi-sound source judging method and device [70] \\
\hline 6 & Mapping & Proactive telephone number mapping configuration management [38] \\
\hline 7 & Planning & Robotic capability model for artificial intelligence assisted manufacturing supply chain planning [37] \\
\hline 8 & Forecasting & Systems and methods for forecasting client needs using interactive communication [55] \\
\hline 9 & Simulation & Method and apparatus for creating a computer simulation of an actor [53] \\
\hline 10 & Recognition & Medical image identification and interpretation [60] \\
\hline B & \multicolumn{2}{|c|}{ PERSONALIZATION } \\
\hline 11 & Adaptive & Adaptive real-time conversational systems and methods [12] \\
\hline 12 & Customization & Client-server hybrid AI scores for customized actions [67] \\
\hline 13 & Dynamic & Dynamically manipulating an emoticon or avatar [13] \\
\hline 14 & Learning & Self learning method and system for managing a group reward system [50] \\
\hline 15 & Natural & Natural interactive user interface using artificial intelligence and freeform input [28] \\
\hline 16 & Notification & Artificial intelligence for resolution and notification of a fault detected by information technology fault monitoring [46] \\
\hline 17 & Interaction & Natural interactive user interface using artificial intelligence and freeform input [28] \\
\hline 18 & Personalizing & Method for personalizing chat bots [69] \\
\hline 19 & Recommending & Method and system for video and film recommendation [11] \\
\hline
\end{tabular}




\begin{tabular}{|c|c|c|}
\hline 20 & Real Time & Method and system for real time visualization of individual health condition on a mobile device [54] \\
\hline $\mathbf{C}$ & PRODUCTIVITY & \\
\hline 21 & Automation & Systems and methods for automatically measuring a video game difficulty [2] \\
\hline 22 & Autonomous & Autonomous information technology diagnostic checks [9] \\
\hline 23 & Assisted & Artificial intelligence assisted tagging of users in digital media online [21] \\
\hline 24 & Classification & Classification technique for multi-band raster data for sorting and processing of colorized data for display [57] \\
\hline 25 & Communication & $\begin{array}{l}\text { Methods and apparatus for performing machine learning to improve capabilities of an artificial intelligence (AI) entity } \\
\text { used for online communications [68] }\end{array}$ \\
\hline 26 & Detection & Mood detection with intelligence agents [6] \\
\hline 27 & Extraction & Utilizing artificial intelligence for data extraction [42] \\
\hline 28 & Generation & System and method for automatic generation of user-oriented homepage [65] \\
\hline 29 & Managing & Digital healthcare information management [58] \\
\hline 30 & Manipulating & Dynamically manipulating an emoticon or avatar [13] \\
\hline 31 & Measuring & Systems and methods for automatically measuring a video game difficulty [2] \\
\hline 32 & Optimizing & Machine-learning systems and techniques to optimize teleoperation and/or planner decisions [39] \\
\hline 33 & Reconstruction & iRecon: intelligent image reconstruction system with anticipatory execution [64] \\
\hline 34 & Regulating & Regulating the growth of complexity in developmental systems [61] \\
\hline 35 & Scheduling & Computerized system and method for distributed energy resource scheduling [51] \\
\hline 36 & Testing & Utilizing artificial intelligence to test cloud applications [63] \\
\hline $\mathbf{D}$ & SECURITY & \\
\hline 37 & Warning & Motor vehicle artificial intelligence expert system dangerous driving warning and control system and method [52] \\
\hline 38 & Monitoring & Monitoring social media for specific issues [47] \\
\hline 39 & Security & Method, system, and computer program product for assessing information security [5] \\
\hline 40 & Tracking & Systems and methods for tracking motion and gesture of heads and eyes [49] \\
\hline
\end{tabular}

\section{Case Studies}

Two case studies were employed to explore the feasibility of the design heuristics - a smart canteen and a smart online shopping system. The smart canteen can be seen as a design brief for digital innovation and the smart online shopping system can be seen as a UX design brief. A novice UX designer was invited to attend the preliminary evaluation study and was asked to generate as many concepts as possible within 20 minutes by utilizing AIDHS 40 including Analogical words and related AI examples (see Table 2) for each predefined design brief. The UX designer was provided extra 15 minutes for writing textual descriptions on each design brief for further analysis.

The case studies were performed by the first author and are original designs to illustrate the heuristics, i.e. they are not based on other case studies or specific existing products. Below are the ideas proposed by the UX designer, using different AIDHS. Two design works are described below with the DHS utilized numbers identified in brackets by \#).

\subsection{Case study: Smart Canteen}

Decision Making. The 'Smart Canteen' can analyze (\#1 Analyzing), judge (\#5 fudging) and forecast (\#8 Forecasting) the peak-hour real-time by monitoring the canteen's environment (\#20 Real-time; \#38 Monitoring). It can give notifications to potential customers for helping them to avoid the peak-hour, thus reducing the waiting time. The 'Smart Canteen' can also help chefs to determine whether they need to cook more dishes (\#2 Determining), potentially reducing the waste of food. AI-based shopping satisfaction evaluation (\#4 Evaluation) can be achieved by detecting users' facial moods (\#26 Detection). Additional concepts include 'mapping the most favorite dishes smartly' (\#06 Mapping), and 'recognizing the dishes for helping staff to calculate the bills productively' (\#10 Recognition).

Personalization. The 'Smart Canteen' provides personalized functions for meeting users' needs. The App for 'Smart Canteen' can recommend dishes to the customer based on their shopping histories (\#19 Recommending). Customers can interact with smart robots for ordering (\#17 Interaction), (e.g., multilingual communication supports).

Productivity. Prices are generated and managed smartly based on AI (\#28 Generation, \#29 Managing) (i.e. dynamic price generation based on the dynamic costs and time factors, etc) (\#13 Dynamic). Optimizing the dishes' making process intelligently (\#32 Optimizing), such as scheduling the dishes marking material procurement smartly (\#35 Scheduling).

Security. Comprehensive management for food security based on AI. For example, monitoring the whole process of raw material for food safety (\#38 Monitoring; \#39 Security) and food traceability (\#40 Tracing). When ordered dishes are lacking in the canteen, the AI will give warnings (\#37 Warning).

\subsection{Case study: Smart Online Shopping System}

Decision Making. AI will assist the customer to evaluate the goods/products comprehensively (\#4 Evaluation; \#23 Assisted) and judge the products' quality (i.e. if it is a fake or low-quality product) (\#5 Fudging). The ‘Smart Online Shopping System’ can analyze the customer's shopping habits automatically (\#1 Analyzing; \#21 Automation) for helping the customer to determine which one is more suitable (\#2 Determining). The 'Smart Online Shopping System' offers 3D simulation service for augmented reality (\#9 Simulation) (e.g. customers can see the fitting effects based on their body size and the type of clothes).

Personalization. Adaptive user interface (UI) (\#11 Adaptive) for enhancing UX based on different platforms (i.e. Smartphone, laptop, and PC). Dynamic product information recommendations for customers (\#13 Dynamic; \#19 Recommending). The system will give the 
customer notifications (\#16 Notification) on Sales and new season products. Natural and interactive customer service assistant, i.e. voice communication for order customers and gesture communication for deaf and hard-of-hearing customers (\#15 Natural; \#17 Interactive; \#25 Communication). The 'smart system' offers personalized/interesting products recommendation to different customers (\#18 Personalization; \#19 Recommending).

Productivity. It also offers the function of key information extraction based on the users' customized definition (\#27 Extraction; \#12 Customization). Smart video generation is based on sorting out goods' pictures and texts for improving users' understanding and attention (\#28 Generation). Managing the 'shopping cart' or 'collection' system intelligently (i.e. combining similar goods in the same category for managing and classifying productivity) (\#29 Managing; \#24 Classification). Optimizing the display of goods' images and textual descriptions by AI for improving users' understanding and attention (\#32 Optimizing). The user interface of the shopping system can differ for different customers (e.g. larger textual display for older customers).

Security. 'Smart Online Shopping System' also offers functions for security such as monitoring the whole process of shopping (\#38 Monitoring; \#39 Security) and risk traceability (\#40 Tracing). Pre-warning will be given when critical issues are detected for risk management and control (\#37 Warning; 26\# Detection).

\section{Discussion}

\subsection{Contributions and implications}

\subsubsection{Design Heuristics for AI.}

Through analyzing 1,755 AI patents (from 2008-2020) granted in the US, China and the Europe (US, CN, EP) based on a four-step analysis method, forty AIDHS were extracted. They were initially evaluated through two case studies. In the case of the smart canteen, 12 ideas were generated and 20 unique heuristics employed. In the case of the smart online shopping system, 15 ideas were generated and 25 unique heuristics employed. Between the two case studies, 31 out of 40 heuristics were utilized. Interestingly, $56 \%$ of ideas employed multiple DHS ( $\geq 2$ ) (e.g. case study 1: 6 out of 12; case study 2: 9 out of 15). The high usage rate of DHS indicates that the AIDHS tool is easy to understand and employ. The 40 AIDHS have the potential to be a design stimuli tool in the early conceptual design phase for helping practitioners to explore a larger design space and generate more AI-powered ideas. More ideas will feed higher quality ideas.

\subsubsection{A New Method for Design Heuristics Extraction.}

Designers face many challenges. For example, they have to learn new knowledge and skills for emerging technologies because new technologies can support designers in the generation of more innovative products [41]. In this paper, we propose a new method for extracting DHS (i.e., Inspirational Design Stimuli) for emerging technologies. The AIDHS will aid practitioners in the ideation stage.

\subsection{Limitations and future work}

AIDHS can help practitioners to generate more AI-powered ideas by opening-up a wider design space when utilizing one or multiple DHS simultaneously. In the later design phase, these concepts may need to be further discussed with AI algorithm scientists/engineers for evaluating technical feasibilities or to reference AI design guidelines for aiding evaluation [3]. These extracted DHS are based on the current state of AI which is a fast-developing technology. Different application scenarios may stimulate new innovations over time.

Future research is needed to validate the effectiveness by utilizing varied metrics for further evaluation (i.e. diversity, usefulness, novelty, and creativity) through a controlled experiment. Future user studies with both novice and expert designers need to be conducted to explore the applicability of the method.

\section{Conclusion}

We have extracted 40 design heuristics for AI through studying 1,755 granted AI patents. The feasibility of the heuristics was preliminarily tested using two AI-powered design briefs - a smart canteen and an online smart shopping system. Results suggest that the $40 \mathrm{AI}$ design heuristics have the potential to support concept generation in the early design phase. AIDHS has proved effective in aiding a novice UX designer in generating large numbers of innovative ideas during the early conceptual design phase. The originality of our study is twofold: 1) we have used a new method to extract design heuristics for AI. 2) we have developed the novel tool AIDHS which can support ideation.

Overall, our study suggests that AIDHS can be employed as a design stimuli tool for ideation. It can help practitioners to explore a larger design space for generating AI-powered ideas, with reduced design fixation [34, 66].

\section{ACKNOWLEDGMENTS}

This research is supported by China Scholarship Council (Grant no: 201906360310).

\section{REFERENCES}

[1] Abou Shousha M, Elsawy ASM. 2019. Artificial intelligence-based system and methods for corneal diagnosis, U.S. Patent No. 10,468,142. 
[2] Aghdaie N, Kolen J, Mattar MM, Sardari M, Xue S, Zaman KA-U, Wu M. 2019. Systems and methods for automatically measuring a video game difficulty, U.S. Patent No. $10,384,133$.

[3] Amershi S, Weld D, Vorvoreanu M, Fourney A, Nushi B, Collisson P, Suh J, Iqbal S, Bennett PN, Inkpen K. 2019. Guidelines for human-AI interaction. Proceedings of the 2019 chi conference on human factors in computing systems, 1-13.

[4] Amico A, Prabhushankar M. 2018. Artificial intelligence based vehicle dashboard analysis, U.S. Patent No. 10,152,641

[5] Baggett Jr CC, Adams JJ. 2005. Method, system and computer program product for assessing information security, U.S. Patent No. 6,925,443.

[6] Baughman AK, Pandey D, Perrino JP, Whitman TR. 2019. Mood detection with intelligence agents, U.S. Patent No. $10,223,641$.

[7] Baxter K. 2019. How to Meet User Expectations for Artificial Intelligence. [online] Medium, Accessed 15.04.2020, 2020, https://medium.com/salesforce-ux/how-to-meetuser-expectations-for-artificial-intelligence-a51d3c82af6.

[8] Bazeley P, Jackson K. 2013. Qualitative data analysis with NVivo (SAGE publications limited).

[9] Bikumala S, Machado CL. 2019. Autonomous information technology diagnostic checks, U.S. Patent No. 10,289,522.

[10] Bloesch-Paidosh A, Shea K. 2019. Design Heuristics for Additive Manufacturing Validated Through a User Study. Journal of Mechanical Design 141(4).

[11] Bonet AT, Marcos JS, Llopis AT, Rivas MGL. 2011. Method and system for video and film recommendation, U.S. Patent No. 8,063,295.

[12] Brown HA, Shelton JK, Ouellette J, Saluja K. 2019. Adaptive real-time conversational systems and methods, U.S. Patent No. 10,440,181.

[13] Brown RW, Hung MHK, Young RL. 2013. Dynamically manipulating an emoticon or avatar, U.S. Patent No. 8,620,850.

[14] Bruseberg A, McDonagh-Philp D. 2002. Focus groups to support the industrial/product designer: a review based on current literature and designers' feedback. Applied Ergonomics 33(1):27-38.

[15] Corbett E, Saul N, Pirrung M. 2018. Interactive machine learning heuristics. Proceedings of the Machine Learning from User Interaction for Visualization and Analytics Workshop at IEEE VIS.

[16] Cross N. 2019. Editorial: Design as a discipline. Design Studies 65:1-5.

[17] Daniel Castro MMaEC. 2019. Who Is Winning the AI Race: China, the EU or the United States? , Accessed 15.04.2020, 2020, https://www.datainnovation.org/2019/08/whois-winning-the-ai-race-china-the-eu-or-the-united-states/.

[18] Dove G, Halskov K, Forlizzi J, Zimmerman J. 2017. UX design innovation: Challenges for working with machine learning as a design material. Proceedings of the 2017 chi conference on human factors in computing systems, 278-288.

[19] Eberle R. 1971. Scamper: games for imagination development. BuffaloN. YDOK.

[20] Edhlund B, McDougall A. 2019. Nvivo 12 Essentials (Lulu. com).

[21] Frigon MF. 2019. Artificial intelligence assisted tagging of users in digital media online, U.S. Patent No. 10,417,275.

[22] Fu K, Moreno D, Yang M, Wood KL. 2014. Bio-Inspired Design: An Overview Investigating Open Questions From the Broader Field of Design-by-Analogy. Journal of Mechanical Design 136(11).

[23] Fu KK, Yang MC, Wood KL. 2016. Design Principles: Literature Review, Analysis, and Future Directions. Journal of Mechanical Design 138(10).

[24] Gadd K. 2011. TRIZ for engineers: enabling inventive problem solving (John wiley \& sons).

[25] Gentner D. 1983. Structure-mapping: A theoretical framework for analogy. Cognitive science 7(2):155-170.

[26] Google. 2019. People + AI Guidebook. [online] People + AI Research team Accessed 15.04.2020, 2020, https://pair.withgoogle.com/chapter/user-needs/.

[27] Guangxu L. 2019. A video evaluation recommendation system, China Patent No. CN109005431B.

[28] Hoetzer J, Miseldine P. 2019. Natural interactive user interface using artificial intelligence and freeform input, U.S. Patent No. 10,402,740.

[29] Howard TJ. 2008. Information management for creative stimuli in engineering design. University of Bath.

[30] Howard TJ, Culley S, Dekoninck EA. 2011. Reuse of ideas and concepts for creative stimuli in engineering design. Journal of Engineering Design $22(8): 565-581$.

[31] Huang C, Notten A, Rasters N. 2011. Nanoscience and technology publications and patents: a review of social science studies and search strategies. Journal of Technology Transfer 36(2):145-172.

[32] Hwang D, Park W. 2018. Design heuristics set for X: A design aid for assistive product concept generation. Design Studies 58:89-126.

[33] Ilevbare IM, Probert D, Phaal R. 2013. A review of TRIZ, and its benefits and challenges in practice. Technovation 33(2-3):30-37.

[34] Jansson DG, Smith SM. 1991. Design fixation. Design studies 12(1):3-11.

[35] Jin X, Dong H. 2020. New design heuristics in the digital era. Proceedings of the Design Society: DESIGN Conference (Cambridge University Press), 607-616.

[36] Jin X, Dong H, Mark E. 2020. New Design Heuristics Compared with Existing Ones. Proceedings of DRS 2020 (Design Research Society \& Griffith University), 1597-1610.

[37] Kohlhepp CA. 2019. Robotic capability model for artificial intelligence assisted manufacturing supply chain planning, U.S. Patent No. $10,242,316$.

[38] Ku B. 2013. Proactive telephone number mapping configuration management, U.S. Patent No. 8,363,815.

[39] Levinson JS, Sibley GT, Rege AG. 2017. Machine-learning systems and techniques to optimize teleoperation and/or planner decisions, U.S. Patent No. 9,632,502.

[40] Linsey JS, Markman AB, Wood KL. 2012. Design by Analogy: A Study of the WordTree Method for Problem Re-Representation. Journal of Mechanical Design 134(4).

[41] Louridas P. 1999. Design as bricolage: anthropology meets design thinking. Design Studies 20(6):517-535.

[42] Magro AMH, Carvajal HB. 2019. Utilizing artificial intelligence for data extraction, U.S. Patent No. 10,223,640.

[43] Mcclanahan CJ, Soss J. 2009. Method for determining acceptability of proposed color solution using an artificial intelligence model, U.S. Patent No. 7,536,231.

[44] Moore A. 2019. When AI Becomes an Everyday Technology. Havard Business Review, Accessed September 12, 2020, 2020, https://hbr.org/2019/06/when-ai-becomes-aneveryday-technology.

[45] Moreno DP, Hernandez AA, Yang MC, Otto KN, Hölttä-Otto K, Linsey JS, Wood KL, Linden A. 2014. Fundamental studies in Design-by-Analogy: A focus on domainknowledge experts and applications to transactional design problems. Design Studies 35(3):232-272.

[46] Mowry E, Ilitch N, Goodrich M, Putans C, Youngs R. 2018. Artificial intelligence for resolution and notification of a fault detected by information technology fault monitoring, U.S. Patent No. $10,162,699$.

[47] Mukherjee R. 2017. Monitoring social media for specific issues, U.S. Patent No. 9,602,575.

[48] Murphy J, Fu K, Otto K, Yang M, Jensen D, Wood K. 2014. Function Based Design-by-Analogy: A Functional Vector Approach to Analogical Search. Journal of Mechanical Design 136(10).

[49] Nie X. 2017. Systems and methods for tracking motion and gesture of heads and eyes, U.S. Patent No. 9,785,249.

[50] Otto J, Van Luchene A. 2012. Self learning method and system for managing a group reward system, U.S. Patent No. 8,224,760. 
[51] Pande SA, Dromey IR. 2016. Computerized system and method for distributed energy resource scheduling, U.S. Patent No. 9,373,960.

[52] Pedersen RD. 2018. Motor vehicle artificial intelligence expert system dangerous driving warning and control system and method, U.S. Patent No. 9,919,648.

[53] Perlin K. 2012. Method and apparatus for creating a computer simulation of an actor, U.S. Patent No. 8,243,078.

[54] Rath MW, Niedzwiecki A, Karnath DF. 2015. Method and system for real time visualization of individual health condition on a mobile device, U.S. Patent No. 9,101,334.

[55] Ross G, Walker T. 2019. Systems and methods for forecasting client needs using interactive communication, U.S. Patent No. 10,275,802.

[56] Sampaio PGV, González MOA, de Vasconcelos RM, dos Santos MAT, de Toledo JC, Pereira JPP. 2018. Photovoltaic technologies: Mapping from patent analysis. Renewable and Sustainable Energy Reviews 93:215-224.

[57] Santos AD, Brandt PH. 2019. Classification technique for multi-band raster data for sorting and processing of colorized data for display, U.S. Patent No. 10,394,883.

[58] Shusterman V. 2010. Digital healthcare information management, U.S. Patent No. 7,801,591.

[59] Simon HA. 1996. The sciences of the artificial (MIT press).

[60] Sorenson JL, MacCutcheon DW, Zhao T, Kuhn G. 2019. Medical image identification and interpretation, U.S. Patent No. 10,452,813.

[61] Steels L. 2008. Regulating the growth of complexity in developmental systems, U.S. Patent No. 7,440,927.

[62] Stone P, Brooks R, Brynjolfsson E, Calo R, Etzioni O, Hager G, Hirschberg J, Kalyanakrishnan S, Kamar E, Kraus S. 2016. Artificial Intelligence and Life in 2030. One hundred year study on artificial intelligence: Report of the 2015-2016 Study Panel. Stanford University, Stanford, CA, http://ailo0. stanford. edu/2016-report. Accessed: September 6:2016.

[63] Takawale P, Dhumal DG, Gupta G, Patidar MD. 2019. Utilizing artificial intelligence to test cloud applications, U.S. Patent No. 10,515,002.

[64] Tung C-H, Dwivedi S, Zhu Y-M, Collins JP. 2019. iRecon: intelligent image reconstruction system with anticipatory execution, U.S. Patent No. $10,275,906$.

[65] Tzeng J-N. 2010. System and method for automatic generation of user-oriented homepage, U.S. Patent No. 7,836,005.

[66] Vasconcelos LA, Crilly N. 2016. Inspiration and fixation: Questions, methods, findings, and challenges. Design Studies 42:1-32.

[67] Vijayant A, Jagota AK. 2019. Client-server hybrid AI scores for customized actions, U.S. Patent No. 10,373,046.

[68] Vora DV, Youngjun K. 2019. Methods and apparatus for performing machine learning to improve capabilities of an artificial intelligence (AI) entity used for online communications, U.S. Patent No. 10,474,673.

[69] Wallace RS. 2014. Method for personalizing chat bots, U.S. Patent No. 8,818,926.

[70] Xinyu W. 2015. Based on artificial intelligence of the intelligent robot of multi-sound source judging method and device, China Patent CN105096956B.

[71] Yang Q. 2018. Machine learning as a UX design material: How can we imagine beyond automation, recommenders, and reminders? 2018 AAAI spring symposium series.

[72] Yang Q, Steinfeld A, Rosé C, Zimmerman J. 2020. Re-examining Whether, Why, and How Human-AI Interaction Is Uniquely Difficult to Design. Proceedings of the 2020 chi conference on human factors in computing systems, 1-13.

[73] Yilmaz S, Seifert C, Daly SR, Gonzalez R. 2016. Design Heuristics in Innovative Products. Journal of Mechanical Design 138(7).

[74] Zhou Z, Sun L, Zhang Y, Liu X, Gong Q. 2020. ML Lifecycle Canvas: Designing Machine Learning-Empowered UX with Material Lifecycle Thinking. Human-Computer Interaction: $1-25$

\section{A APPENDICES}

\section{A.1 Preliminary DHS Extraction Based on the Word Frequency and researchers' analysis}

\begin{tabular}{|c|c|c|c|c|c|c|c|c|c|}
\hline LEARNING & 76 & SIMULATION & 44 & AUTONOMOUS & 29 & IDENTIFYING & 21 & SCHEDULING & 5 \\
\hline MANAGING & 70 & RECOGNITION & 43 & PREDICTION & 29 & MEASURING & 17 & WARNING & 5 \\
\hline ANALYSIS & 65 & MONITORING & 42 & ADAPTIVE & 27 & PLANS & 17 & JUDGING & 3 \\
\hline DETECTION & 59 & DIAGNOSIS & 38 & NATURAL & 25 & NOTIFICATION & 16 & MANIPULATING & 3 \\
\hline COMMUNICATION & 54 & AUTOMATED & 37 & TRACKING & 24 & ANALYZING & 14 & RECONSTRUCTION & 3 \\
\hline INTERACTIVE & 52 & SECURITY & 33 & CLASSIFICATION & 23 & FORECASTING & 14 & REGULATING & 3 \\
\hline AUTOMATIC & 47 & IDENTIFICATION & 31 & TESTING & 23 & EVALUATION & 12 & & \\
\hline DYNAMIC & 46 & DETERMINING & 30 & CONFIGURING & 22 & CUSTOMIZED & 11 & & \\
\hline GENERATION & 46 & PERSONALIZING & 30 & DECISION & 22 & MAPPING & 11 & & \\
\hline OPTIMIZING & 45 & ASSISTED & 29 & RECOMMENDING & 22 & EXTRACTION & 7 & & \\
\hline
\end{tabular}

